\title{
Unsupervised Active Learning of CRF Model for Cross-Lingual Named Entity Recognition
}

\author{
Mohamed Farouk Abdel Hady, Abubakrelsedik Karali, Eslam Kamal, \\ and Rania Ibrahim \\ Microsoft Research \\ Cairo, Egypt \\ mohabdel@microsoft.com
}

\begin{abstract}
Manual annotation of the training data of information extraction models is a time consuming and expensive process but necessary for the building of information extraction systems. Active learning has been proven to be effective in reducing manual annotation efforts for supervised learning tasks where a human judge is asked to annotate the most informative examples with respect to a given model. However, in most cases reliable human judges are not available for all languages. In this paper, we propose a cross-lingual unsupervised active learning paradigm (XLADA) that generates high-quality automatically annotated training data from a word-aligned parallel corpus. To evaluate our paradigm, we applied XLADA on English-French and English-Chinese bilingual corpora then we trained French and Chinese information extraction models. The experimental results show that XLADA can produce effective models without manually-annotated training data.
\end{abstract}

Keywords: Information extraction, named entity recognition, crosslingual domain adaptation, unsupervised active learning.

\section{Introduction}

Named Entity Recognition (NER) is an information extraction task that identifies the names of locations, persons, organizations and other named entities in text, which plays an important role in many Natural Language Processing (NLP) applications such as information retrieval and machine translation. Numerous supervised machine learning algorithms such as Maximum Entropy, Hidden Markov Model and Conditional Random Field (CRF) [1 have been adopted for NER and achieved high accuracy. They usually require large amount of manually annotated training examples. However, it is time-consuming and expensive to obtain labeled data to train supervised models. Moreover, in sequence modeling like NER task, it is more difficult to obtain labeled training data since hand-labeling individual words and word boundaries is really complex and need professional annotators. Hence, the shortage of annotated corpora is the obstacle of supervised learning and limits the further development, especially for languages for which such resources are scarce. Active learning is the method which, 
instead of relying on random sampling from the large amount of unlabeled data, it reduces the cost of labeling by actively participates in the selection of the most informative training examples then an oracle is asked for labeling the selected sample. There are two settings depending on the oracle type: supervised setting 2] where requires human annotators as oracle for manual annotation and the unsupervised setting where the oracle is an automation process. Using different settings, active learning may determine much smaller and most informative subset from the unlabeled data pool. The difference between unsupervised active learning and semi-supervised learning [3] is that the former depends on an oracle to automatically annotate the most informative examples with respect to the underlying model. The later depends on the underlying model to automatically annotate some unlabeled data, to alleviate mislabeling noise the model selects the most confident examples.

For language-dependent tasks such as information extraction, to avoid the expensive re-labeling process for each individual language, cross-lingual adaptation, is a special case of domain adaptation, refers to the transfer of classification knowledge from one source language to another target language.

In this paper, we present a framework for incorporating unsupervised active learning in the cross-lingual domain adaptation paradigm (XLADA) that learns from labeled data in a source language and unlabeled data in the target language. The motivation of $X L A D A$ is to collect large-scale training data and to train an information extraction model in a target language without manual annotation but with the help of an effective information extraction system in a source language, bilingual corpus and word-level alignment model.

\section{Related Work}

Yarowsky et al. 4] used word alignment on parallel corpora to induce several text analysis tools from English to other languages for which such resources are scarce. An NE tagger was transferred from English to French and achieved good classification accuracy. However, Chinese NER is more difficult than French and word alignment between Chinese and English is also more complex because of the difference between the two languages.

Some approaches have exploited Wikipedia as external resource to generate NE tagged corpus. Kim et al. [5] build on prior work utilizing Wikipedia metadata and show how to effectively combine the weak annotations stemming from Wikipedia metadata with information obtained through English-foreign language parallel Wikipedia sentences. The combination is achieved using a novel semi-CRF model for foreign sentence tagging. The model outperforms both standard annotation projection methods and methods based solely on Wikipedia metadata. XLADAdoes not leverage Wikipedia because its content is poor in some languages like Chinese.

Fu et al. 6] presents an approach to generate large-scale Chinese NER training data from an English-Chinese discourse level aligned parallel corpus. It first employs a high performance NER system on one side of a bilingual corpus. 
And then, it projects the NE labels to the other side according to the word level alignment. At last, it selects labeled sentences using different strategies and generate an NER training corpus. This approach can be considered as passive domain adaptation while $X L A D A$ is active learning framework that filters out the auto-labeled data and selects the most informative training sentences.

Muslea et al. [7] introduced Co-Testing, a multi-view active learning framework, where two models are trained on two independent and sufficient sets of features. The most informative sentences are the points of disagreement between the two models that could improve their performance and a human judge is asked for labeling them. On the other hand, XLADA looks for the most informative sentences for the target model and we don't have judges.

Jones et al. 3] adapted semi-supervised learning Co-EM to information extraction tasks to learn from both labeled and unlabeled data that makes use of two distinct feature sets (training document's noun phrases and context). It is interleaved in the supervised active learning framework Co-Testing. XLADA differs in that cross-lingual label propagation on a parallel corpus is interleaved for automatic annotation instead of using Co-EM approach and that it adopts an unsupervised active learning strategy.

$X L A D A$ is more practical than the framework proposed by $\mathrm{Li}$ et al. 8 that depends on cross-lingual features extracted from the word-aligned sentence pair in training the target language CRF model. Hence, it isn't possible to extract named entities from a sentence in the target language unless it is aligned with a sentence in the source language.

\section{Algorithmic Overview}

The architecture of the proposed combination of cross-lingual domain adaptation and active learning paradigm $X L A D A$ is shown in Figure 1.

\subsection{Initial Labeling}

Source Language NER. An effective source language NER is applied on the source-side of the bilingual corpus $U_{S}$ to identify named entities such as person, location, organization names, denote the output $L_{S}$. In our experiments, the source language is English and English Stanford NER ${ }^{1}$ is used. The system is based on linear chain CRF 1] sequence models that can recognize three types of named entities (Location, Person and Organization).

Word Alignment of Parallel Corpus. Sentence alignment and word alignment is performed on the given unlabeled bilingual corpus $U_{S}$ and $U_{T}$. First, sentence level alignment is performed then we applied word dependent transition model based HMM (WDHMM) for word alignment [9].

\footnotetext{
${ }^{1}$ http://nlp.stanford.edu/software/CRF-NER.shtml
} 


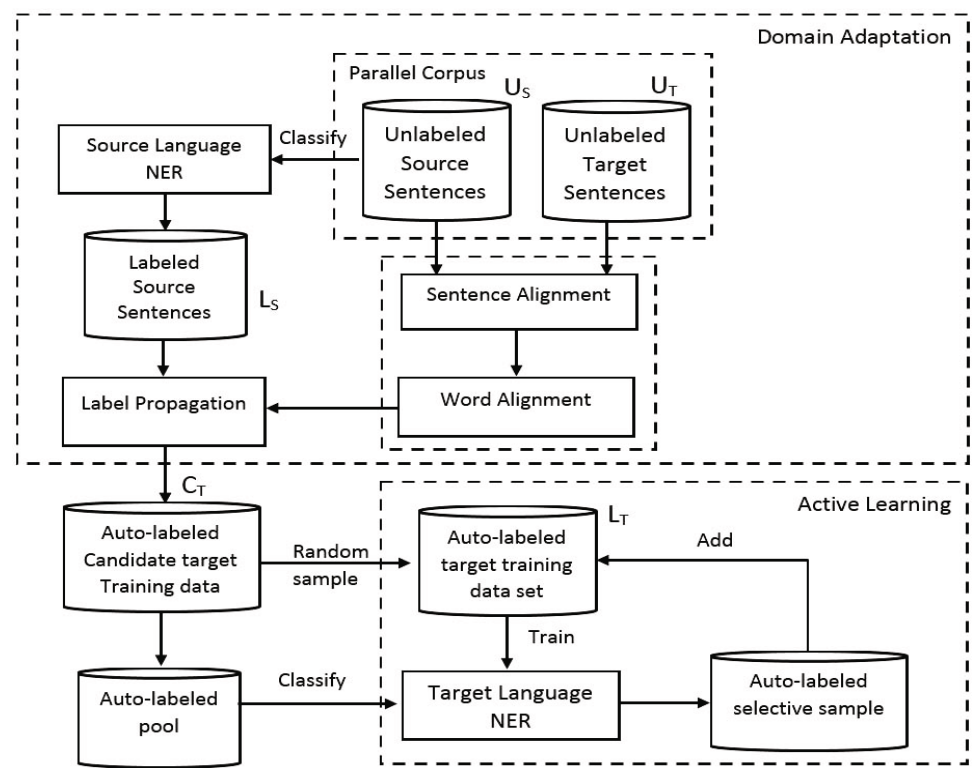

Fig. 1. Architecture of cross-lingual active domain adaptation (XLADA)

Label Propagation. We project the NE labels to the target side of the parallel corpus to automatically annotate target language sentences, according to the result of word alignment, as shown in Figure 2 The output is a set of candidate training sentences. A target sentence is filtered out from the set of candidate training sentences if the number of named entities after label propagation is less than the number of named entities in the source sentence.

\subsection{Unsupervised Active Learning}

The amount of auto-labeled sentences in the target language training is too huge to be used for training the information extraction model. Also they are noisy because of the errors in source language NER or word-level alignment. Unsupervised active learning is adopted for selecting high quality training sentences used to train CRF model. The manual annotation of the selected sentences by human judges is replaced with the alignment-based automatic annotation.

We randomly select a set of auto-labeled training sentences $L_{T}$. An initial CRF model is trained with $L_{T}$. Since a random set of auto-labeled sentences is not sufficient to train a good prediction model in the target language, additional labeled data is required to reach a reasonable prediction model. Afterward, $X L A D A$ will proceed in an iterative manner.

A pool $C P_{T}$ of the large amount of auto-labeled sentences is selected. There are two ways to select the sentences in the pool, either a random sample or by assigning a score for each target sentence and finally choose sentences with the highest score (most confident sentences). 


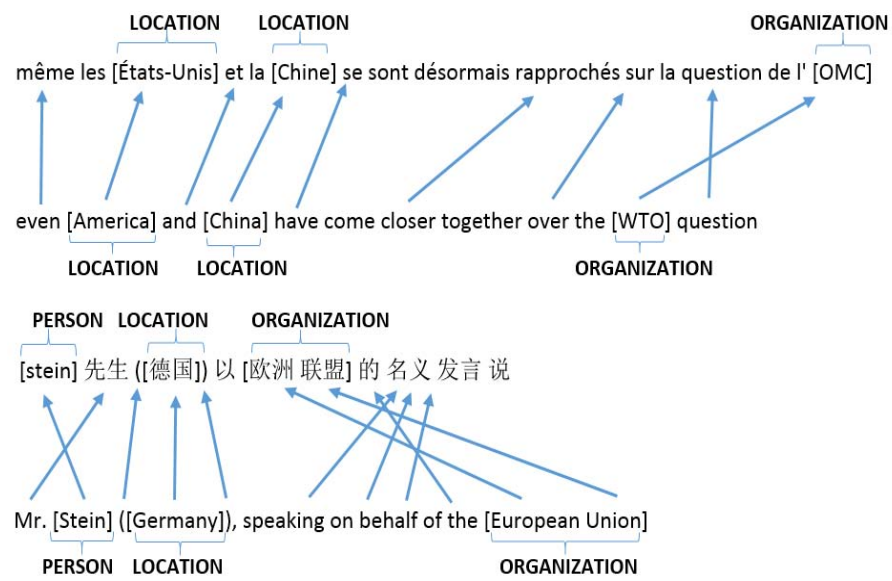

Fig. 2. Projection of named-entity tags from English to Chinese and French sentences

The score of each target sentence depends on the score given to its corresponding source sentence in the parallel corpus, as follows:

$$
\operatorname{score}(S)=\min _{w_{i} \in S} \max _{c_{j} \in \text { classes }} P\left(c_{j} \mid w_{i}, \theta_{\text {src }}\right)
$$

The source NER model $\theta_{s r c}$ assigns probability for each token of how likely it belongs to each entity type: person, location, organization or otherwise. Then, the entity type for each token is the class with maximum probability $P\left(c_{j} \mid w_{i}, \theta_{\text {src }}\right)$. We apply the forward-backward algorithm to compute them.

In each round of active learning, the current target NER model $\theta_{\text {tgt }}$ tags each target sentence in the auto-labeled pool $C P_{T}$. The critical challenge lies in how to select the most informative sentences for labeling. Based on different measurements of target sentence informativeness, we propose the following metric to measure how informative is a given sentence $S$.

$$
i n f \operatorname{orm}(S)=\frac{1}{N(S)} \sum_{w_{i} \in S} \hat{y}\left(w_{i}\right) P\left(\hat{y}_{t g t}\left(w_{i}\right) \mid w_{i}, \theta_{t g t}\right)
$$

where $\hat{y}\left(w_{i}\right)=I\left(\hat{y}_{s r c}\left(w_{i}\right) \neq \hat{y}_{\text {tgt }}\left(w_{i}\right)\right)$ the indicator boolean function between

$$
\hat{y}_{s r c}\left(w_{i}\right)=\arg \max _{c_{j} \in \text { classes }} P\left(c_{j} \mid w_{i}, \theta_{\text {src }}\right)
$$

the NE label propagated from the source NER model $\theta_{\text {src }}$ through alignment information and

$$
\hat{y}_{t g t}\left(w_{i}\right)=\arg \max _{c_{j} \in \text { classes }} P\left(c_{j} \mid w_{i}, \theta_{\text {tgt }}\right)
$$

the NE tag assigned by the current target NER model $\theta_{\text {tgt }}$ to the $i^{\text {th }}$ word in $S$.

The most informative sentences are the ones that the target NER model $\theta_{t g t}$ didn't learn yet (least confident on its NE prediction) and mismatch with the 
source NER model $\theta_{\text {src }}$ where $N(S)$ is the number of tokens in $S$ the two models disagree. Then we select the top $N$ sentences or the ones less than a predefined threshold, add them to $L_{T}$ with the automatic labels propagated from the source NER model $\theta_{\text {src }}$ and remove them from the pool $C P_{T}$. After new labels being acquired, the target model is retrained on the updated $L_{T}$.

\subsection{Conditional Random Field}

Conditional Random Fields (CRFs) [10], similar to the Hidden Markov Models (HMMs) [1], are a type of statistical modeling method used for labeling or parsing of sequential data, such as natural language text and computer vision. $\mathrm{CRF}$ is a discriminative undirected probabilistic graphical model that calculates the conditional probability of output values for a given observation sequence. HMMs made strong independence assumption between observation variables, in order to reduce complexity, which hurts the accuracy of the model while CRF does not make assumptions on the dependencies among observation variables.

Figure 3 shows the graphical representation of liner chain CRFs. Because of its linear structure, linear chain $\mathrm{CRF}$ is frequently used in natural language processing to predict sequence of labels $Y$ for a given observation sequence $X$. The inference of a linear-chain CRF model is that given an observation sequence $X$, we want to find the most likely sequence of labels $Y$. The probability of $Y$ given $X$ is calculated as follows:

$$
P(Y \mid X)=\frac{1}{Z(X)} \exp \left(\sum_{t=1}^{T} \sum_{i=1}^{n} w_{i} f_{i}\left(y_{t-1}, y_{t}, X, t\right)\right)
$$

where

$$
Z(X)=\sum_{Y^{\prime}} \exp \left(\sum_{t=1}^{T} \sum_{i=1}^{n} w_{i} f_{i}\left(y_{t-1}^{\prime}, y_{t}^{\prime}, X, t\right)\right)
$$

In the equation, the observation sequence $X=\left(x_{1}, \ldots, x_{T}\right)$, the label sequence $Y=\left(y_{1}, \ldots, y_{T}\right)$ where $y_{t}$ is the label for position $t$, state feature function is concerned with the entire observation sequence, the transition feature between labels of position $t-1$ and $t$ on the observation sequence is also considered. Each feature $f_{i}$ can either be state feature function or transition feature function. The coefficients $w_{i}$ s are the weights of features and can be estimated from training data. $Z(X)$ is a normalization factor.

\section{Experiments}

\subsection{Datasets}

The performance of $X L A D A$ is evaluated on the unsupervised learning of Chinese and French NER for named entity recognition of three entity types, person (PER), location (LOC) and organization (ORG). To achieve this goal, unlabeled 


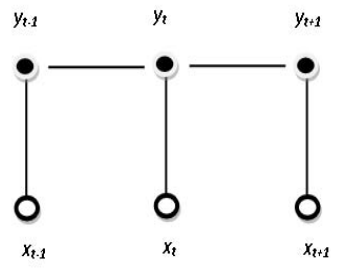

Fig. 3. Graphical representation of linear-chain CRF

training data set and labeled test data set is required for each target language. As unlabeled training data, two bilingual parallel corpora is used. The EnglishChinese corpus is 20 million parallel sentences and the English-French corpus contains 40 million parallel sentences. The corpora involve a variety of publicly available data sets including United Nations proceeding 2 , proceedings of the European Parliament3, Canadian Hansards 4 and web crawled data. Both sides of each corpus were segmented (in Chinese) and tokenized (in English and French).

Table 1. Corpora used for performance evaluation

\begin{tabular}{|l|c|c|}
\hline test set & Chinese & French \\
\hline \#sentences & 5,633 & 9,988 \\
\hline \#Person & 2,807 & 3,065 \\
\hline \#Location & 7,079 & 3,153 \\
\hline \#Organization & 3,827 & 1,935 \\
\hline
\end{tabular}

Table 1 shows a description of the corpora used as labeled test data for $X L A D A$. One is the Chinese OntoNotes Release 2.0 corpus 5 and the second is a French corpus manually labeled using crowd sourcing. A group of five human annotators was asked to label each sentence then the majority NE tag is assigned to each token.

\subsection{Setup}

A widely used open-source NER system, Stanford Named Entity Recognizer is employed to detect named entities in the English side of the English-Chinese and English-French parallel corpora.The number of sentences that has at least one named entity detected by the Stanford NER is around 4 million sentences for Chinese and 10 million sentences for French. The features used to train the CRF model are shown in Figure 2. It's worth mentioning that the trainer used here is a local implementation of CRF (not Stanford's implementation) since Stanford's implementation is very slow and memory consuming.

\footnotetext{
${ }^{2}$ http://catalog.1dc.upenn.edu/LDC94T4A

3 http://www.statmt.org/europarl/

4 http://www.isi.edu/natural-language/download/hansard/

5 http://catalog.ldc.upenn.edu/LDC2008T04
} 
Table 2. Features used for Named Entity Recognition CRF model

\begin{tabular}{|c|l|}
\hline type & extracted features \\
\hline Shape Features & $\begin{array}{l}\text { WordString, WordShape, StartsWithCapital, AllCapital } \\
\text { Character N-Grams, Shape Character N-Grams }\end{array}$ \\
\hline Brown Clusters 12 & Levels 0, 4, 8, 12 \\
\hline Bags of Words & Date Tokens, Punctuations, Personal Titles, Stop Words \\
\hline Contextual & Previous 3 words and next 2 word features \\
\hline
\end{tabular}

As baselines for comparison, we have studied the following data selection techniques:

- random sample: The first NER model was trained on randomly sample of 340,000 and 400,000 sentences from the four million auto-labeled sentences and ten million sentences for Chinese and French language, respectively. (upper horizontal dashed lines in Figure 4)

- most confident sample: the second NER model was trained on the set of the top 340,000 and the top 400,000 most confident sentences (based on the min-max score function defined on section 3) for Chinese and French, respectively. (lower horizontal dashed lines in Figure 4)

For active learning, we have randomly chosen 100,000 auto-labeled sentences to train the initial NER for Chinese and French, respectively. And then, we have created a pool(set) of two million sentences where we have two experiments:

- random pool: one with a pool of randomly chosen sentences regardless of tagging confidence.

- most confident pool: another experiment with a pool of target sentences corresponding to the most confident source sentences selected by min-max score function.

The initial NER is applied on the pool and the informativeness of each sentence is measured using the function defined in section 3 .

- informative addition: The most informative sentences are the sentences with score less than 0.9. At the end of the first iteration, the labeled training set is augmented with the newly-selected most informative sentences and the target NER is re-trained, this process is repeated for 20 iterations where the final NER for Chinese and French has been trained on 340,000 sentences and 400,000 sentences, respectively.

- random addition: another baseline for comparison where in each iteration, a number of auto-labeled sentences in the target language, Chinese or French, is randomly selected, equals to the number of most informative sentences selected in the same iteration at the informative addition experiment.

\subsection{Results}

The performance of unsupervised Chinese and French NER systems is reported in Table 4 and Table 3 , respectively where the best performing data selection 
Table 3. The performance of unsupervised French NER models trained using XLADA compared to baselines

\begin{tabular}{|c|c|c|c|c|c|c|c|}
\hline \multirow{3}{*}{\begin{tabular}{|c} 
\\
Entity \\
type
\end{tabular}} & \multirow{3}{*}{\begin{tabular}{|c}
$\begin{array}{c}\text { selection } \\
\text { method }\end{array}$ \\
$\begin{array}{c}\text { Pool } \\
\text { type }\end{array}$
\end{tabular}} & \multirow{2}{*}{\multicolumn{2}{|c|}{$\begin{array}{c}\text { XLADA } \\
\text { informative addition }\end{array}$}} & \multicolumn{4}{|c|}{ Baseline } \\
\hline & & & & \multicolumn{2}{|c|}{ random addition } & \multirow{2}{*}{$\begin{array}{c}\text { most } \\
\text { confident } \\
\text { sample }\end{array}$} & \multirow{2}{*}{$\begin{array}{l}\text { random } \\
\text { sample }\end{array}$} \\
\hline & & \begin{tabular}{|c|} 
most \\
confident
\end{tabular} & random & \begin{tabular}{|c|} 
most \\
confident
\end{tabular} & random & & \\
\hline \multirow{3}{*}{ PER } & Precision & 78.1 & 79.7 & 78.6 & 79.0 & 77.9 & 77.0 \\
\hline & Recall & 82.0 & 79.6 & 78.8 & 75.4 & 71.9 & 76.2 \\
\hline & F1 & 80.0 & 79.7 & 78.7 & 77.1 & 74.7 & 76.6 \\
\hline \multirow{3}{*}{ LOC } & Precision & 82.9 & 82.9 & 83.2 & 83.8 & 73.0 & 84.6 \\
\hline & Recall & 65.9 & 66.6 & 64.0 & 64.9 & 59.2 & 63.8 \\
\hline & F1 & 73.4 & 73.9 & 72.3 & 73.2 & 65.4 & 72.7 \\
\hline \multirow{3}{*}{ ORG } & Precision & 54.1 & 50.1 & 52.0 & 51.7 & 59.1 & 49.5 \\
\hline & Recall & 50.0 & 52.2 & 48.7 & 49.6 & 25.0 & 48.1 \\
\hline & F1 & 51.9 & 51.1 & 50.3 & 50.6 & 35.2 & 48.8 \\
\hline
\end{tabular}

technique is bold faced. Figure 4 shows the learning curve of target NER models using the different training data selection techniques for Chinese and French, respectively. The F1 measure of both random sample NER and most confident sample NER is drawn as a horizontal dashed line.The results show that XLADA outperforms the random sample baseline.

For Chinese NER. For person NE, XLADA with informative addition using most confident pool achieves the highest F1-score $80.4 \%$ compared to $59.5 \%$ for most confident sample and $75.1 \%$ for random sample. This is attributed to the increase in person recall from $43.6 \%$ and $63.2 \%$ to $69.7 \%$ and $68.0 \%$ respectively. For location NE, XLADA with informative addition using most confident pool achieves the highest F1-score $83.1 \%$ compared to $73.3 \%$ for most confident sample and $81.7 \%$ for random sample. This is attributed to the increase in location recall from $64.6 \%$ and $74.0 \%$ to $76.4 \%$ and $75.0 \%$ respectively. For organization NE, $X L A D A$ with informative addition using random pool achieves the highest F1score $65.9 \%$ compared to $44.5 \%$ for most confident sample and $62.6 \%$ for random sample. This is attributed to the increase in organization recall from $29.4 \%$ and $50.3 \%$ to $55.2 \%$, respectively.

For French NER. For person NE, XLADA with informative addition using most confident pool achieves the highest F1-score $80.0 \%$ compared to most confident sample with $74.7 \%$ and random sample with $76.6 \%$. This is attributed to the increase in person recall from $71.9 \%$ and $76.2 \%$ to $82.0 \%$. For location NE, XLADA with informative addition using random pool achieves the highest F1-score $73.9 \%$ compared to domain adaptation without active learning: most confident sample of $65.4 \%$ and random sample of $72.7 \%$. This is attributed to the increase in location recall from $59.2 \%$ and $63.8 \%$ to $66.6 \%$. For organization NE, $X L A D A$ with informative addition using most confident pool achieves the highest 
Table 4. The performance of unsupervised Chinese NER models trained using XLADA compared to baselines

\begin{tabular}{|c|c|c|c|c|c|c|c|}
\hline \multirow{3}{*}{\begin{tabular}{|c} 
\\
Entity \\
type
\end{tabular}} & \multirow{3}{*}{$\begin{array}{c}\begin{array}{c}\text { selection } \\
\text { method }\end{array} \\
\begin{array}{c}\text { Pool } \\
\text { type }\end{array}\end{array}$} & \multicolumn{2}{|c|}{ XLADA } & \multicolumn{4}{|c|}{ Baseline } \\
\hline & & \multicolumn{2}{|c|}{ informative addition } & \multicolumn{2}{|c|}{ random addition } & \multirow{2}{*}{$\begin{array}{c}\text { most } \\
\text { confident } \\
\text { sample }\end{array}$} & \multirow{2}{*}{$\begin{array}{l}\text { random } \\
\text { sample }\end{array}$} \\
\hline & & \begin{tabular}{|c|} 
most \\
confident
\end{tabular} & random & \begin{tabular}{|c|} 
most \\
confident
\end{tabular} & random & & \\
\hline \multirow{3}{*}{ PER } & Precision & 94.9 & 93.5 & 92.9 & 91.8 & 93.4 & 92.4 \\
\hline & Recall & 69.7 & 68.0 & 62.7 & 65.0 & 43.6 & 63.2 \\
\hline & F1 & 80.4 & 78.8 & 74.9 & 76.1 & 59.5 & 75.1 \\
\hline \multirow{3}{*}{$\mathrm{LOC}$} & Precision & 91.1 & 92.2 & 90.9 & 91.1 & 84.9 & 91.1 \\
\hline & Recall & 76.4 & 75.0 & 73.6 & 73.9 & 64.6 & 74.0 \\
\hline & F1 & 83.1 & 82.7 & 81.3 & 81.6 & 73.3 & 81.7 \\
\hline \multirow{3}{*}{ ORG } & Precision & 80.8 & 81.7 & 87.2 & 78.6 & 91.4 & 83.0 \\
\hline & Recall & 51.9 & 55.2 & 47.7 & 51.6 & 29.4 & 50.3 \\
\hline & F1 & 63.2 & 65.9 & 61.6 & 62.3 & 44.5 & 62.6 \\
\hline
\end{tabular}

F1-score $51.9 \%$ compared to most confident sample of $35.2 \%$ and random sample of $48.8 \%$. This is attributed to the significant improvement of organization recall from $25.0 \%$ and $48.1 \%$ to $50.0 \%$.

\subsection{Discussion}

The improvement in recall means increase in the coverage of the trained NER model. This is attributed to the high quality of the training sentences selected by the proposed selective sampling criterion compared to random sampling. In addition, it is better than selecting target sentences where the English NER model is most confident about their corresponding English ones. The reason is that although the English NER model is most confident, this does not alleviate the passive nature of the target NER model as it has no control on the selection of its training data based on its performance. That is, it implies that the selected sentences do not carry new discriminating information with respect to the target NER model. In all cases, the random sample outperforms the most confident sample. The reason that selecting only the most confident sentences tends to narrow the coverage of the constructed NER. Figure 4 shows that XLADA achieves the most significant performance improvement in the early iterations, then the learning curve starts to saturate.

In general, the results of organization NE type are lower than the results of Person and Location. The reason is that ORG names are more complex than Person and Location names. They usually consist of more words, which may result in more word alignment errors and then lead to more training sentences being filtered out. Another reason behind this is that ORG names mostly consist of a combination of common words. gNot only for French and Chinese but also English ORG entity recognition is more difficult, which also results in more noise among the ORG training sentences. 


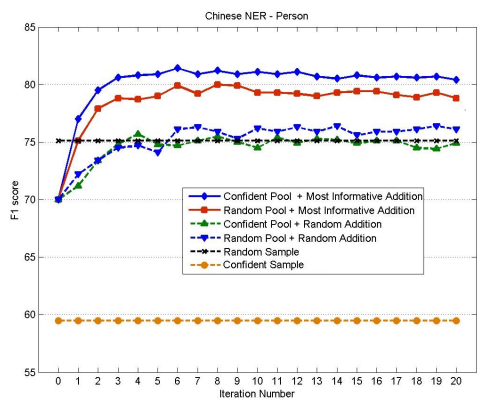

(a) chinese person

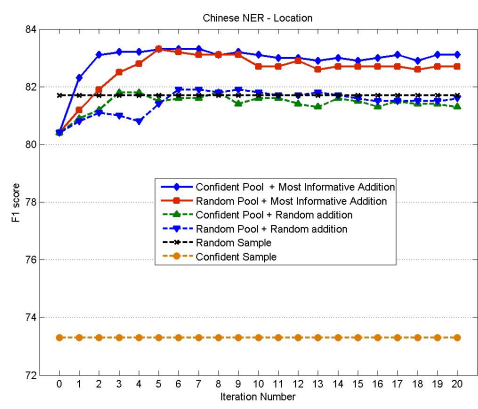

(c) chinese location

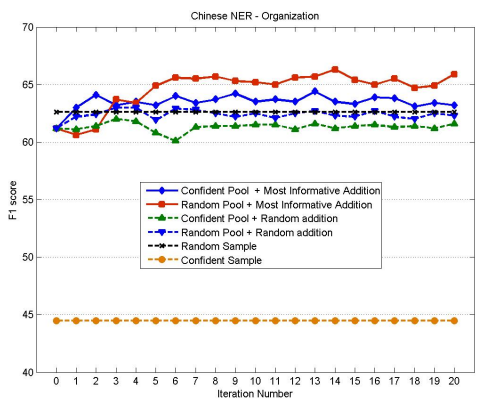

(e) chinese organization

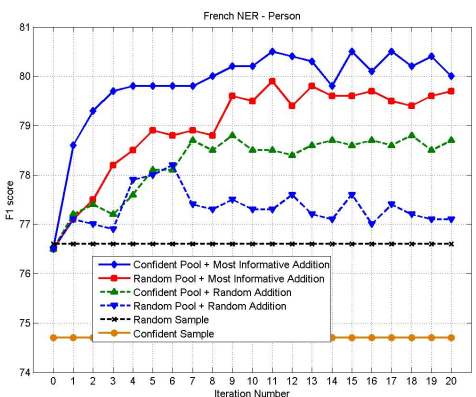

(b) french person

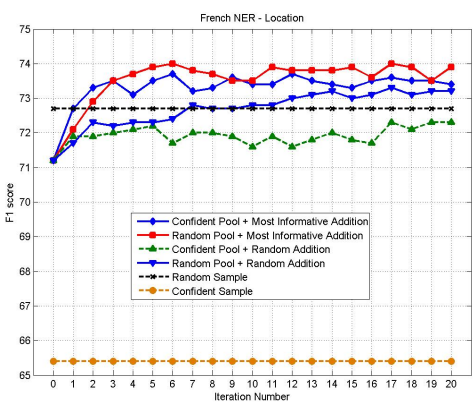

(d) french location

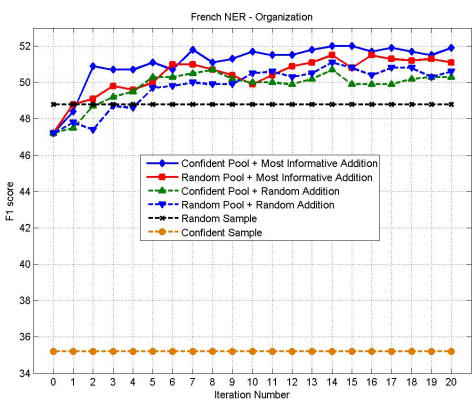

(f) french organization

Fig. 4. Performance of unsupervised Chinese and French NER

\section{Conclusions}

The manual annotation of training sentences to build an information extraction system for each language is expensive, error-prone and time consuming. We introduced an unsupervised variant of active learning in the cross-lingual automatic annotation framework that replaces the manual annotation with the 
alignment-based automatic annotation. It depends on the existence of high quality source language NER model, bilingual parallel corpus and word-level alignment model. A modified score function is proposed as the criterion for selecting the most informative training sentences from the huge amount of automatically annotated sentences. Although the reported results are on the recognition of three entity types, the framework can be generalized to any information extraction task.

\section{References}

1. McCallum, A., Li, W.: Early results for named entity recognition with conditional random fields, feature induction and web-enhanced lexicons. In: Proceedings of CoNLL (2003)

2. Esuli, A., Marcheggiani, D., Sebastiani, F.: Sentence-based active learning strategies for information extraction. In: Proceedings of the 2nd Italian Information Retrieval Workshop (IIR 2010), pp. 41-45 (2010)

3. Jones, R., Ghani, R., Mitchell, T., Rilo, E.: Active learning for information extraction with multiple view. In: Proceedings of the European Conference in Machine Learning (ECML 2003), vol. 77, pp. 257-286 (2003)

4. Yarowsky, D., Ngai, G., Wicentowski, R.: Inducing multilingual text analysis tools via robust projection across aligned corpora. In: Human Language Technology Conference, pp. 109-116 (2001)

5. Kim, S., Toutanova, K., Yu, H.: Multilingual named entity recognition using parallel data and metadata from Wikipedia. In: Proceedings of the 50th Annual Meeting of the Association for Computational Linguistics (2012)

6. Fu, R., Qin, B., Liu, T.: Generating chinese named entity data from a parallel corpus. In: Proceedings of the 5th International Joint Conference on Natural Language Processing, pp. 264-272 (2011)

7. Muslea, I., Minton, S., Knoblock, C.A.: Active learning with multiple views. Journal of Artificial Intelligence Research 27, 203-233 (2006)

8. Li, Q., Li, H., Ji, H.: Joint bilingual name tagging for parallel corpora. In: Proceedings of CIKM 2012 (2012)

9. He, X.: Using word-dependent transition models in HMM based word alignment for statistical machine translation. In: Proceedings of the Second Workshop on SMT (WMT). Association for Computational Linguistics (2007)

10. Lafferty, J., McCallum, A., Pereira, F.: Conditional random fields: Probabilistic models for segmenting and labeling sequence data. In: Proceedings of the International Conference on Machine Learning (ICML), pp. 282-289 (2001)

11. Rabiner, L.: A tutorial on hidden markov models and selected applications in speech recognition. Proceedings of the IEEE 77, 257-286 (1989)

12. Brown, P.F., de Souza, P.V., Mercer, R.L., Pietra, V.J.D., Lai, J.C.: Class-based n-gram models of natural language. Computational Linguistics 18(4) (1992) 